\title{
Fogitare
}

\section{CONDUTAS QUE PODEM INTERFERIR NA FASE PRÉ- ANALÍTICA DO EXAME SUMÁRIO DE URINA}

\author{
Suzana Carvalho de Sousa Pedrosa ${ }^{1}$ (i) \\ Milenna Azevedo Minhaqui Ferreira ${ }^{2}$ (1) \\ Keyth Sulamitta Lima Guimarães ${ }^{3}$ (1) \\ Wilma Ferreira Guedes Guimarães ${ }^{3}$ (1) \\ Keylla Talitha Fernandes Barbosa ${ }^{3}$ (1)
}

\section{RESUMO}

Objetivo: identificar as condutas pré-analíticas que podem influenciar nos resultados do exame sumário de urina.

Método: trata-se de um estudo exploratório, descritivo, do tipo transversal, de natureza quantitativa, realizada com 246 pacientes de um laboratório público de referência do município de João Pessoa, Paraíba, durante os meses de junho e julho de 2019. A análise de dados foi realizada por estatística descritiva e inferencial, com Teste de Qui-Quadrado e Correlação de Spearman, elencado após o teste de Kolmogorov-Sminorv.

Resultados: em relação ao conhecimento sobre o exame, $138(56,1 \%)$ dos participantes afirmaram possuí-lo, porém $174(70,7 \%)$ relataram não terem sido orientados antes da coleta do exame. A correlação evidenciou que, quanto maior o conhecimento, mais adequadas eram as condutas préanalíticas.

Conclusão: observou-se baixo nível de conhecimento sobre o procedimento, contribuindo para condutas pré-analíticas inadequadas. Destaca-se a importância de ofertar ao cliente orientações sobre a coleta do exame.

DESCRITORES: Enfermagem; Técnicas de Laboratório Clínico; Coleta de Urina; Conhecimento; Testes Diagnósticos de Rotina.

\section{CONDUCTAS QUE PUEDEN INTERFERIR EN LA FASE PREANALÍTICA DE LA PRUEBA SUMARIA DE ORINA}

\section{RESUMEN:}

Objetivo: identificar las condiciones preanalíticas que pueden influir en los resultados de la prueba sumaria de orina. Método: se trata de un estudio exploratorio, descriptivo, transversal, de carácter cuantitativo, realizado con 246 pacientes de un laboratorio público de referencia del municipio de João Pessoa, Paraíba, durante los meses de junio y julio de 2019. El análisis de los datos se realizó mediante estadísticas descriptivas e inferenciales, con la prueba de Chi-cuadrado y la correlación de Spearman, que figuran después de la prueba de Kolmogorov-Smirnov. Resultados: en cuanto a los conocimientos sobre el examen, $138(56,1 \%)$ de los participantes afirmaron tenerlos, pero $174(70,7 \%)$ declararon no haber recibido orientación antes del examen. La correlación demostró que cuanto mayor era el conocimiento, más adecuados eran los procedimientos preanalíticos. Conclusión: se observó un bajo nivel de conocimiento sobre el procedimiento, lo que contribuyó a que las conclusiones preanalíticas fueran inadecuadas. Destaca la importancia de ofrecer al cliente orientaciones sobre la coleta de la prueba.

DESCRIPTORES: Enfermería; Técnicas de Laboratorio Clínico; Toma de Muestras de Orina; Conocimiento; Pruebas Diagnósticas de Rutina. 
Atualmente, o objetivo mais importante da medicina diagnóstica é garantir aos profissionais da saúde e pacientes um atendimento eficiente e seguro, fornecendo resultados com precisão absoluta, sejam laboratoriais ou de imagem, com resultados confiáveis para posterior tomada de decisão dos médicos em relação à conduta clínica dos seus pacientes. As falhas nessa análise são ameaças significativas para a segurança dos pacientes, acarretando atraso e ainda a falta de diagnóstico(1).

Para melhor compreender as fontes de erros em laboratórios clínicos, primeiro devese conhecer e analisar as fases e os processos que compõem as técnicas diagnósticas. Teoricamente, as fases laboratoriais dividem-se classicamente em três: pré-analítica, analítica e pós-analítica. A fase pré-analítica está relacionada com a solicitação da análise, passando pela orientação relevante dos pacientes, coleta, identificação, armazenamento, transporte e recebimento das amostras biológicas. Estimam-se que problemas nessa etapa sejam responsáveis por cerca de $70 \%$ dos erros ocorridos nos laboratórios ${ }^{(2)}$.

Diversos materiais biológicos podem ser analisados, tais como sangue, urina, fezes, líquido cefalorraquidiano, exsudato vaginal, anal, orofaringe, ocular e de outras regiões do corpo, dentre outros tipos de amostras ${ }^{(3)}$. O exame sumário de urina é composto por um conjunto de características, sendo as de aspectos físico-químicos: volume, aspecto, cor, cheiro, densidade e $\mathrm{pH}$, proteínas, hemoglobinas, glicose, cetonas, bilirrubinas, urobilinogênio, nitrito e leucócitos. Entre os elementos presentes no sedimento urinário, temos: filamentos de muco, células epiteliais, leucócitos, hemácias, cilindro, cristais, bactérias de fermentação e outros elementos. Faz-se importante a análise conjunta de todos esses elementos para a definição clínica e terapêutica do paciente ${ }^{(4)}$.

Os tipos de amostras mais utilizados para a realização do sumário de urina são: aleatória, que é realizada a qualquer momento do dia, desde que respeitado o intervalo de duas horas após a micção; a primeira urina da manhã, considerada a amostra ideal para análise por estar mais concentrada e apresentar resultados mais fidedignos; e, por fim, a segunda urina da manhã, que deve ser coletada entre duas e quatro horas após a primeira micção, período em que o paciente deve permanecer em jejum ${ }^{(4-5)}$.

A análise do sumário de urina é de grande relevância para auxiliar no diagnóstico e acompanhamento de distintas patologias como: infecções renais e das vias urinárias, doenças metabólicas não relacionadas com o sistema urinário, como diabetes mellitus, doenças da tireoide ou de fígado, bem como o rastreamento do exame clínico de rotina antes da internação hospitalar e antes de procedimentos cirúrgicos ${ }^{(5)}$.

É reconhecido que os principais interferentes na análise do sedimento urinário são a contaminação da coleta ou a falta de experiência do analista, tanto por microscopia óptica comum quanto por métodos automatizados. Isto mostra o quanto é importante a coleta adequada do material a ser examinado(4).

Destaca-se que a fase pré-analítica se inicia na coleta de material, realizada pelo paciente, que deve ser informado de todas as condutas preconizadas, seja no ambiente domiciliar ou hospitalar. O erro nesta etapa varia desde o descuido e a falta de orientação ao paciente, até a ausência de entendimento sobre boas práticas em laboratório, bem como treinamento ineficiente. A adequada gestão desses processos pré-analíticos garante a precisão dos resultados da fase analítica ${ }^{(2)}$.

Justifica-se o presente estudo visto que determinadas condutas podem comprometer o resultado da análise laboratorial e, dessa forma, afetar o tratamento e as condutas clínicas estabelecidas para o paciente. Resultados falsos negativos podem culminar em danos irreparáveis ao cliente, uma vez que as decisões terapêuticas são instituídas com base no diagnóstico. Diante da problemática apresentada, o presente artigo teve como 
objetivo identificar as condutas pré-analíticas que podem influenciar nos resultados do exame sumário de urina.

\section{MÉTODO}

Estudo descritivo, transversal de natureza quantitativa, realizado em um laboratório público de referência do município de João Pessoa-PB, Brasil. O referido serviço realiza exames de baixa, média e alta complexidade, auxiliando os profissionais da saúde na prevenção e tratamento das doenças.

A população do estudo foi composta por 710 pacientes, que realizaram o exame de sumário de urina no ano que precedeu a pesquisa. Posteriormente, delimitou-se a amostra considerando a seguinte fórmula: $n=Z 2 P Q / d 2$, sendo $n=$ tamanho amostral mínimo; $Z=$ variável reduzida; $P=$ probabilidade de encontrar o fenômeno estudado; $Q=1-P$; $\mathrm{d}=$ precisão desejada. Adotou-se $\mathrm{p}=50 \%$, por se tratar de uma avaliação multidimensional, e parâmetro de erro amostral de $5 \%$. Por meio do referido cálculo, obteve-se um tamanho amostral mínimo de 240 indivíduos.

Foram incluídos no estudo indivíduos de ambos os sexos, com idade igual ou superior a 18 anos, que possuíam capacidade de comunicação verbal e estivessem com amostra para realização do sumário de urina. Considerou-se inelegíveis aqueles pacientes que iriam fazer outros tipos de exame de urina, como a urocultura. Frente à disponibilidade dos participantes e considerando os critérios de elegibilidade ora descritos, a amostra final foi composta por 246 clientes.

Os dados foram coletados de junho a julho de 2019, com um instrumento estruturado contendo questões sociodemográficas e as características da amostra do sumário de urina, como local e profissional que solicitou o exame, assim como as características da coleta, a exemplo do ambiente em que foi coletada a amostra, o tempo entre a coleta e a entrega no laboratório, o coletor utilizado, se realizou a higiene íntima antes da coleta e se recebeu orientações acerca do exame, assim como o nível de conhecimento sobre a temática.

A análise dos dados se deu por meio da estatística descritiva de natureza univariada para todas as variáveis, incluindo medidas de frequência, de posição e dispersão. Para comparação das principais variáveis categóricas, foi utilizado o teste Qui-quadrado, de acordo com os objetivos propostos para o estudo. A fim de verificar a normalidade dos dados quantitativos, adotou-se o teste de Kolmogorov-Smirnov. Em seguida, utilizou-se - Coeficiente de Correlação de Spearman, considerando o nível de significância de 5\%. Para tanto, elencou-se o sistema computacional Statistical Package for the Social Sciences - SPSS versão 22.0.

É oportuno ressaltar que a pesquisa seguiu os princípios éticos estabelecidos na Resolução n466/2012 do Conselho Nacional de Saúde que diz respeito às Diretrizes e Normas Regulamentadoras de Pesquisa Envolvendo Seres Humanos. O Projeto de pesquisa foi aprovado pelo Comitê de Ética em Pesquisa do Centro Universitário de João Pessoa conforme parecer 3.351.230.

Foram avaliados 246 pacientes, desses $202(82,1 \%)$ eram do sexo feminino enquanto apenas $44(17,9 \%)$ pertenciam ao sexo masculino. Em relação ao estado civil, verificou-se 
que 137 (55,7\%) eram casados, 130 (52,8\%) autodeclarados pardos e 108 (43,9\%) com ensino fundamental completo. Ademais, 140 (56,9\%) dos entrevistados referiram seguir os preceitos católicos e a maioria residia no município de João Pessoa $(n=243 ; 98,8 \%)$, conforme evidenciado na Tabela 1.

Tabela 1 - Descrição dos dados sociodemográficos. João Pessoa, PB, Brasil, 2019

\begin{tabular}{|c|c|c|c|}
\hline Variável & Categorias & $\mathbf{n}$ & $\%$ \\
\hline \multirow{2}{*}{ Sexo } & Feminino & 202 & 82,1 \\
\hline & Masculino & 44 & 17,9 \\
\hline \multirow{5}{*}{ Estado civil } & Casado & 137 & 55,7 \\
\hline & Solteiro & 67 & 27,2 \\
\hline & Divorciado & 17 & 6,9 \\
\hline & Viúva & 24 & 9,8 \\
\hline & Não respondeu & 1 & 0,4 \\
\hline \multirow{4}{*}{ Raça/Cor } & Parda & 130 & 52,8 \\
\hline & Branca & 98 & 39,8 \\
\hline & Preta & 17 & 6,9 \\
\hline & Indígena & 1 & 0,4 \\
\hline \multirow{4}{*}{ Escolaridade } & Analfabeto & 24 & 9,8 \\
\hline & Ensino Fundamental & 108 & 43,9 \\
\hline & Ensino Médio & 105 & 42,7 \\
\hline & Ensino Superior & 9 & 3,7 \\
\hline \multirow{5}{*}{ Religião } & Católico & 140 & 56,9 \\
\hline & Evangélico & 83 & 33,7 \\
\hline & Espírita & 3 & 1,2 \\
\hline & Outros & 5 & 2 \\
\hline & Ignorado & 15 & 6,1 \\
\hline \multirow{2}{*}{$\begin{array}{l}\text { Município de } \\
\text { residência }\end{array}$} & João Pessoa & 243 & 98,8 \\
\hline & Interior & 3 & 1,2 \\
\hline
\end{tabular}

Fonte: Autores (2019)

Considerando os dados dispostos na Tabela 2, os exames de urina foram, em sua maioria, solicitados nas Unidades Básicas de Saúde da Família, com 180 (73,2\%) dos casos relatados, em que o profissional médico foi o principal responsável pela solicitação $(74,4 \%)$. Entre os participantes do estudo, 235 (95,5\%) afirmaram que coletaram a primeira amostra, sendo 232 (94,3\%) em seu domicílio. Identificou-se também que o tempo entre a coleta e a entrega da amostra no laboratório especializado durou, em média, de duas a três horas em 155 (63\%) casos. Ademais, 243 (98,8\%) pacientes informaram ter utilizado o coletor estéril para o armazenamento da amostra, enquanto $152(61,8 \%)$ dos indivíduos confirmam ter realizado higiene íntima antes da coleta. 
Tabela 2 - Descrição dos dados do sumário de urina. João Pessoa, PB, Brasil, 2019

\begin{tabular}{|c|c|c|c|}
\hline Variável & Categorias & $\mathbf{n}$ & $\%$ \\
\hline \multirow{3}{*}{ Local da solicitação } & Atenção Básica & 180 & 73,2 \\
\hline & Clínica & 38 & 15,4 \\
\hline & Hospital & 28 & 11,4 \\
\hline \multirow{2}{*}{ Profissional solicitante } & Médico & 183 & 74,4 \\
\hline & Enfermeiro & 63 & 25,6 \\
\hline \multirow{3}{*}{ Tipo de amostra } & Amostra aleatória & 0 & 0,4 \\
\hline & Primeira amostra & 235 & 95,5 \\
\hline & Segunda amostra & 10 & 4,1 \\
\hline \multirow{2}{*}{ Local coletado } & Residência & 232 & 94,3 \\
\hline & Laboratório & 14 & 5,7 \\
\hline \multirow{3}{*}{ Tempo de coleta } & Até $2 \mathrm{~h}$ & 76 & 30,9 \\
\hline & $2 \mathrm{~h}-3 \mathrm{~h}$ & 155 & 63 \\
\hline & $3 h-4 h$ & 15 & 6,1 \\
\hline \multirow{2}{*}{ Coletor utilizado } & Estéril & 243 & 98,8 \\
\hline & Não estéril & 3 & 1,2 \\
\hline \multirow{2}{*}{ Higiene íntima } & Sim & 152 & 61,8 \\
\hline & Não & 94 & 38,2 \\
\hline
\end{tabular}

Fonte: Autores (2019)

Considerando os dados dispostos na Tabela 3, 117 (52,4\%) confirmam ter colhido a urina a partir do segundo jato. Em relação ao conhecimento sobre o exame, 138 (56,1\%) dos participantes afirmaram possuí-lo, porém 174 (70,7\%) relataram não ter sido orientados previamente à coleta do exame. Daqueles que receberam orientações, 33 (45,8\%) foram informados pelo médico de como deveria ser coletada a amostra de urina.

Tabela 3 - Descrição dos dados do sumário de urina. João Pessoa, PB, Brasil, 2019 (continua)

\begin{tabular}{llcc} 
Variável & Categorias & $\mathbf{n}$ & $\%$ \\
\hline \multirow{2}{*}{ Jato colhido } & Primeiro jato & 117 & 47,6 \\
\cline { 2 - 4 } & Segundo jato & 129 & 52,4 \\
\hline $\begin{array}{l}\text { Possui conhecimento sobre } \\
\text { a coleta de urina }\end{array}$ & Sim & 138 & 56,1 \\
\cline { 2 - 4 } & Não & 108 & 43,9 \\
\hline \multirow{2}{*}{$\begin{array}{l}\text { Orientações prévias } \\
\text { Profissional que forneceu } \\
\text { orientações }\end{array}$} & Não & 174 & 70,7 \\
\cline { 2 - 4 } & Sim & 72 & 29,3 \\
\hline
\end{tabular}


Fonte: Autores (2019)

\begin{tabular}{lcc} 
Enfermeiro & 28 & 38,9 \\
\hline Amigos e familiares & 9 & 12,5 \\
\hline Outros & 2 & 2,8
\end{tabular}

Considerando os dados dispostos na Tabela 4, verificou-se que, entre as mulheres, a maioria referiu possuir conhecimento sobre o exame de urina se comparado com os homens. A escolaridade mostrou uma associação estatisticamente significativa com o nível de conhecimento $(p<0,001)$ : os anos de escolaridade estavam relacionados com a compreensão sobre o tema em questão. As variáveis tempo entre a coleta e a entrega da amostra ao laboratório $(p=0,002)$, higiene íntima $(p<0,001)$ e jato de urina coletado $(p<0,001)$ demonstraram associação estatisticamente significativa com o nível de conhecimento.

Tabela 4 - Associação entre o conhecimento dos pacientes frente ao exame de urina e as características sociodemográficas e coleta de amostra. João Pessoa, PB, Brasil, 2019 (continua)

\begin{tabular}{|c|c|c|c|c|c|}
\hline \multirow[t]{3}{*}{ Variável } & \multicolumn{4}{|c|}{ Possui conhecimento sobre a coleta de urina } & \multirow[t]{3}{*}{$\mathbf{p}$} \\
\hline & \multicolumn{2}{|c|}{ Sim } & \multicolumn{2}{|c|}{ Não } & \\
\hline & $\mathbf{n}$ & $\%$ & $\mathbf{n}$ & $\%$ & \\
\hline \multicolumn{5}{|l|}{ Sexo } & \multirow[t]{4}{*}{$\mathrm{p}<0,001$} \\
\hline Feminino & 125 & 61,9 & 77 & 38,1 & \\
\hline Masculino & 13 & 29,5 & 31 & 70,5 & \\
\hline Total & 138 & 56,1 & 108 & 43,9 & \\
\hline \multicolumn{5}{|l|}{ Escolaridade } & \multirow[t]{6}{*}{$p<0,001 \times$} \\
\hline Analfabeto & 11 & 45,8 & 13 & 54,2 & \\
\hline Ensino Fundamental & 48 & 44,4 & 60 & 55,6 & \\
\hline Ensino Médio & 74 & 70,5 & 31 & 29,5 & \\
\hline Ensino Superior & 5 & 55,6 & 4 & 44,4 & \\
\hline Total & 138 & 56,1 & 108 & 43,9 & \\
\hline \multicolumn{5}{|l|}{ Tempo de coleta } & \multirow[t]{5}{*}{$p=0,002$} \\
\hline Até 2 horas & 49 & 64,5 & 27 & 35,5 & \\
\hline 2 a 3 horas & 85 & 54,8 & 70 & 45,2 & \\
\hline 3 a 4 horas & 4 & 26,7 & 11 & 73,3 & \\
\hline Total & 138 & 56,1 & 108 & 43,9 & \\
\hline \multicolumn{5}{|l|}{ Higiene íntima } & \multirow[t]{4}{*}{$p<0,001^{\star}$} \\
\hline Sim & 116 & 76,3 & 36 & 23,7 & \\
\hline Não & 22 & 23,4 & 72 & 76,6 & \\
\hline Total & 138 & 56,1 & 108 & 43,9 & \\
\hline Jato colhido & & & & & $\mathrm{p}<0,001^{*}$ \\
\hline
\end{tabular}




\begin{tabular}{lcccc}
\hline Primeiro & 32 & 27,4 & 85 & 72,6 \\
\hline Segundo & 106 & 82,2 & 23 & 17,8 \\
\hline Total & 138 & 56,1 & 108 & 43,9
\end{tabular}

* Qui-quadrado

Fonte: Autores (2019)

A correlação entre a média de condutas pré-analíticas corretas e o nível de conhecimento e orientações prévias sobre o exame apresentaram significância estatística $(p \leq 0,05)$, sendo evidenciada uma relação diretamente proporcional, visto que à medida em que as condutas pré-analíticas eram realizadas corretamente, foi possível observar uma maior proporção de conhecimento sobre a temática, conforme Tabela 5.

Tabela 5 - Correlação entre o nível de conhecimento e orientação sobre o exame e as condutas préanalíticas corretas. João Pessoa, PB, Brasil, 2019

\begin{tabular}{lcc} 
Correlação & \multicolumn{2}{c}{ Condutas corretas } \\
\cline { 2 - 3 } & $\mathbf{r}$ & $\mathbf{p}^{*}$ \\
\hline Conhecimento & 0,546 & $\mathrm{p}<0,001$ \\
\hline Orientações & 0,384 & $\mathrm{p}<0,001$ \\
* Coeficiente de Correlação de Spearman & \\
Fonte: Autores (2019) &
\end{tabular}

Entre as fases existentes nos exames laboratoriais, é reconhecido que a etapa préanalítica é responsável por cerca de $46 \%$ a $70 \%$ dos erros. Essa fase se inicia desde a solicitação do exame, preparo do paciente, coleta, manipulação, transporte e armazenamento da amostra, até a entrega do material coletado ao laboratório para análise. Portanto, é necessário que o profissional da saúde tenha conhecimento para que o paciente seja bem instruído, com informações claras sobre a coleta do exame do sumário de urina, uma vez que frequentemente ocorre fora do laboratório ${ }^{(6)}$.

Em relação aos dados sociodemográficos, evidenciou-se o predomínio do sexo feminino, demonstrando uma maior preocupação por parte das mulheres em cuidar da própria saúde, corroborado pela maior procura aos serviços de saúde, se comparado aos homens. Desta forma, os dados observados nessa pesquisa vão ao encontro de um estudo realizado no município de Ribeirão Preto-SP, em que foi revelado que as mulheres buscam os serviços de saúde 1,9 vez mais que os indivíduos do sexo masculino ${ }^{(7)}$.

É reconhecido que as mulheres são as mais suscetíveis a infecções do trato urinário inferior, e diversos fatores podem influenciar a ocorrência deste evento. Destaca-se entre eles o tamanho da uretra da mulher, que é mais curta que a do homem, facilitando a entrada de microrganismos, assim como também a sua maior proximidade com o vestíbulo 
vaginal e com o ânus, o que favorece a colonização pelas bactérias intestinais. Portanto, tais fatores podem levar as mulheres a realizarem mais exames de urina ${ }^{(8)}$.

Verificou-se no presente estudo que a maioria dos entrevistados apresentou baixo nível de escolaridade. O nível de escolaridade está diretamente associado ao entendimento de instruções, sobretudo quanto às condutas a serem seguidas na realização da coleta de exames. Uma pesquisa realizada na Atenção Básica de um município da região Oeste Catarinense corrobora esse achado, ao afirmar que as situações de vulnerabilidade social, como a baixa escolaridade referida pelos usuários e a não compreensão do significado de algumas palavras, e até mesmo o desconhecimento sobre o que é um órgão genital, dificultavam de maneira significante a compreensão do modo como se deve realizar a coleta da amostra urinária ${ }^{(9)}$.

Ademais, verificou-se uma associação estatisticamente significativa entre o nível de escolaridade e o nível de conhecimento sobre a execução do exame. Evidenciou-se que, quanto mais baixo o nível de escolaridade, menos se sabe sobre o preparo para coleta do sumário de urina. Além de ser um componente importante na determinação de hábitos, a escolaridade influencia no modo de obtenção de informações. Indivíduos com alto nível educacional possuem fácil acesso a diferentes meios de informação, como livros, internet e revistas, além de demonstrarem maior capacidade de processar e compreender as informações ${ }^{(10)}$.

No que concerne ao local da solicitação, verificou-se o domínio das requisições de exame do sumário de urina de pacientes advindos da Atenção Primária de Saúde (APS). A APS é considerada a principal porta de entrada da Rede de Atenção à Saúde e ordenadora das ações e cuidados, uma vez que tem papel fundamental para resolução de problemas de menor densidade tecnológica, como a solicitação de exames laboratoriais, atuando também na promoção, prevenção, proteção da saúde e executando ações que proporcionem um cuidado integral aos usuários ${ }^{(11)}$.

Em relação ao profissional solicitante, houve predominância do médico, embora os enfermeiros, principalmente da APS, também tenham liberdade para solicitar tal procedimento, atribuído como rotina estabelecida pelo Ministério da Saúde, e em rotinas aprovadas pelas instituições de saúde. Destarte, é imprescindível que os enfermeiros exerçam com plena capacidade e responsabilidade a compreensão sobre a solicitação e a interpretação dos resultados, associando clinicamente com os sinais e sintomas apresentados pelos pacientes ${ }^{(12)}$.

Observou-se o predomínio da coleta da primeira amostra da manhã entre os entrevistados. A preferência do tipo de amostra depende muito dos exames a serem realizados, contudo frequentemente é orientado a coleta da primeira urina do dia, de maneira a proporcionar mais comodidade ao paciente. Publicações a consideram a amostra ideal para o exame de rotina de urina, por ser mais concentrada e possibilitar a detecção de substâncias químicas e elementos formados, os quais podem estar ausentes em amostras aleatórias ${ }^{(4)}$.

Em relação ao local em que foi realizada a coleta, houve predomínio do ambiente domiciliar. Apesar da facilidade e simplicidade desse exame, é importante que o paciente seja bem orientado quanto às condutas corretas para que o resultado seja o mais fidedigno possível e não altere o diagnóstico. Ademais, dessa forma evita-se a repetição por inutilidade da amostra, o que gera gastos desnecessários tanto para o paciente como para o serviço(6).

Outro cuidado importante é o tempo entre a coleta e a entrega de material ao laboratório. No presente estudo, verificou-se que os usuários entregaram a amostra entre duas a três horas após micção. Caso a análise do material não seja realizada em um prazo máximo de duas horas, a amostra deverá ser refrigerada em caixas isotérmicas. É imprescindível que todos os profissionais de saúde passem essas orientações de maneira concisa, evitando assim que os usuários ultrapassem esse prazo. Destaca-se que o material que ultrapassar esse tempo transcorrido de casa até entrega da amostra ao laboratório, 
certamente sofrerá alterações na fase analítica, resultando em exames imprecisos e com possibilidade de erros ${ }^{(4)}$.

Verificou-se no presente estudo a prevalência do uso de coletores estéreis, que são adequados para o exame sumário de urina. É de grande relevância o uso de recipiente apropriado para a coleta da amostra, o qual precisa ser estéril, ter boca larga para auxiliar o uso por usuários do sexo feminino, ter fundo redondo e amplo para evitar tombamento, além de ser transparente, facilitando a visualização da cor e aspecto da urina(13-14). E indispensável que o enfermeiro e sua equipe forneçam orientações quanto ao uso de coletor apropriado e o manuseio correto, evitando assim a contaminação da amostra.

Foi possível obter na pesquisa dados relevantes relacionados à falta de higiene íntima, que consiste na limpeza da região urogenital com água e sabão. Essa conduta pré-analítica referente ao asseio é primordial, visto que reduz possíveis interferências na urinálise. No estudo realizado no noroeste do estado do Rio Grande do Sul - Brasil, revelou-se que as amostras de urina apresentaram nitrito e aumento na quantidade de bactérias devido à falta de higiene durante a coleta(6).

Ao avaliar a forma em que foi coletada a amostra, evidenciou-se um percentual significativo de usuários que retiram o primeiro jato da urina, configurando-se como conduta inadequada, pois o jato recomendado é o médio, evitando assim erros na fase analítica e confusão na interpretação do resultado final do exame. Estudos demonstram que a coleta do jato médio, ou segundo jato, é de grande relevância, a fim de reduzir contaminantes da uretra e região genitália(6).

Embora tenha se verificado um alto índice de indivíduos que relataram ter recebido orientações prévias, 43,9\% demonstraram conhecimento insuficiente sobre o assunto. Tal fato demonstra que ainda existe um quantitativo significativo de usuários que recebem a requisição do exame e não são orientados corretamente. Pesquisas corroboram o referido achado, ao afirmar as dificuldades vivenciadas no dia a dia, como a sobrecarga de atividades, o não conhecimento da técnica adequada, as limitações frente ao entendimento, bem como a vergonha dos usuários em tirar dúvidas com os profissionais de saúde, o que favorecem a absorção de informações incorretas por meio de amigos e familiares ${ }^{(9-12)}$. Ressalta-se ainda que o profissional que mais forneceu informações foi o médico, justificado pela predominância de exames solicitados por essa especialidade.

Verificou-se também uma associação significativa entre os erros na coleta, como higiene íntima inadequada, tempo entre a coleta e a entrega da amostra prolongado, bem como o jato incorreto, com o conhecimento reduzido sobre o exame laboratorial. As deficiências educacionais favorecem o acesso reduzido às informações e comprometem a execução da assistência, contribuindo para desigualdades no âmbito da saúde e a geração de iniquidades sociais. Verifica-se que o reduzido nível educacional pode afetar negativamente os aspectos socioeconômicos e culturais, além das condições de saúde. Indivíduos que apresentam um menor grau de instrução estão mais expostos a fatores de riscos preveníveis, reafirmando assim a necessidade do ensino como um instrumento de prevenção de agravos e promoção de práticas saudáveis ${ }^{(13)}$.

Portanto, os profissionais devem promover educação em saúde aos usuários, de modo a explicar a importância do manejo adequado para coleta de urina. Ações educativas precisam ser estabelecidas por todos os profissionais que integram a equipe de saúde, principalmente da APS, visto que esse serviço é um dos mais procurados pela população. Desta forma, faz-se necessário adotar práticas para melhoria na hora de solicitar exame do sumário de urina, como o fornecimento de orientações de forma didática, para facilitar o entendimento dos usuários ${ }^{(14)}$.

Diante disso, dentre os profissionais solicitantes, o enfermeiro se destaca como um agente transformador, visto que está mais próximo ao paciente. Logo, consiste em uma das suas atribuições fornecer orientações de maneira adequada, instruindo os usuários sobre o preparo para coleta da amostra a ser analisada ${ }^{(12-14)}$. Desta forma, ressalta-se a 
necessidade em promover educação em saúde, mostrando de forma dinâmica o passo a passo para realização da coleta da urina, com palavras claras e de fácil entendimento, possibilitando a troca de saberes com os usuários, de modo aberto e acolhedor, buscando o esclarecimento de dúvidas e assim fortalecendo o vínculo entre usuário e profissional.

As limitações deste estudo referem-se à escassez deste tema na literatura científica, que dificultou as análises para a discussão dos resultados, sendo necessária a continuação de pesquisas com o tema em questão.

\section{CONCLUSÃO}

Compreende-se que as condutas pré-analíticas inadequadas constatadas no presente estudo, e o desconhecimento quanto ao procedimento a ser seguido para coleta do exame sumário de urina, são resultados da falta de orientação dos profissionais envolvidos, tornando-se preocupante já que pode levar a erros analíticos. Portanto, faz-se necessária a promoção da educação em saúde por meio da participação social, visando à diminuição da vulnerabilidade social de erros ocorridos pelo desconhecimento e, assim, reduzam-se as condutas inadequadas interferente no resultado final da urina, como também os custos relacionados à nova coleta de exame para confirmação de resultados.

Enfatiza-se que este estudo oferece grande contribuição à sociedade no sentido de conhecimento sobre o procedimento de coleta adequada, com construção de estratégias que possibilitem a redução de fatores que interferem negativamente na fase pré-analítica, e aos profissionais de saúde, com ênfase na equipe de enfermagem, no que diz respeito ao fornecimento de informações concisas aos pacientes.

Ressalta-se a importância da solicitação deste exame pelos enfermeiros da atenção básica, visto que isso melhora tanto o entendimento do próprio profissional sobre a clínica do paciente, quanto melhora o fluxo no serviço no que concerne a uma resolução mais rápida de demandas. Além disso, salienta-se a importância no desenvolvimento de atividades com abordagens educativas sobre o processo da coleta de urina.

\section{REFERÊNCIAS}

1. Rosa CDP da, Mathias D, Rovai RL. Sistemas de informação na área da saúde: a informação clínica como instrumento de trabalho para os profissionais de saúde. Polêmica. [Internet]. 2015 [acesso em 23 maio 2020]; 15(2). Disponível em: https://www.e-publicacoes.uerj.br/index.php/polemica/article/ view/17839/13289.

2. Pimenta DZ, Zanusso Júnior G. Principais fatores pré-analíticos interferentes nos exames laboratoriais do coagulograma completo. Rev. Uningá [Internet]. 2016 [acesso em 23 maio 2020]; 25(3). Disponível em: http://revista.uninga.br/index.php/uningareviews/article/view/1780.

3. Souza DS, Carmo RG, Silva DR, Caires DR, Barbiéri RS. Os principais erros na fase pré-analítica em laboratório de análises clínicas. In: 11 Anais CONAC; 2017 Set. p. 7-10; Porto Seguro, Brasil. Bahia: Congresso Nacional de Conhecimento; 2017.

4. Sociedade Brasileira de Patologia Clínica. Fatores pré-analíticos e interferentes em ensaios laboratoriais. São Paulo: Manole; 2018.

5. Fischbach FT, Fischbach MA. Exames laboratoriais e diagnósticos em enfermagem. Guia prático. 6. ed. 
Rio de Janeiro: Guanabara Koogan, 2016.

6. Silva B da, Dal Molin DB, Mendes GA. Adequabilidade de amostras de urina recebidas por um laboratório de análises clínicas do noroeste do estado do Rio Grande do Sul. Rev. Bras. Anal. Clin. [Internet]. 2016 [acesso em 23 maio 2020]; 48(4). Disponível em: http://doi.org/10.21877/24483877.201600491.

7. Levorato CD, Mello LM de, Silva AS da, Nunes AA. Fatores associados à procura por serviços de saúde numa perspectiva relacional de gênero. Ciênc. saúde coletiva. [Internet]. 2014 [acesso em 23 maio 2020]; 19(4). Disponível em: http://dx.doi.org/10.1590/1413-81232014194.01242013.

8. Afonso LR, Wagner R. Exame de urina tipo I em uma comunidade do Bairro Alto - Curitiba - PR. Cad. saúde pública [Internet]. 2017 [acesso em 23 maio 2020]; 1(9). Disponível em: https://portaldeperiodicos. unibrasil.com.br/index.php/cadernossaude/article/view/2378.

9. Engel FD, Metelski FK, Korb A. Orientações para a coleta de urina para exame: desafios que permeiam a atuação da enfermagem. Rev baiana enferm. [Internet]. 2018 [acesso em 23 maio 2020]; 32:e27568. Disponível em: http://dx.doi.org/10.18471/rbe.v32.27568.

10. Nardelli GG, Malaquias BSS, Gaudenci EM, Ledic CS, Azevedo NF, Martins VE, et al. Conhecimento sobre síndrome da imunodeficiência humana de idosos de uma unidade de atenção ao idoso. Rev Gaúcha Enferm. [Internet]. 2016 [acesso em 23 maio 2020]; 37(spe). Disponível em: https://doi.org/10.1590/19831447.2016.esp.2016-0039.

11. Ministério da Saúde. Portaria n. 2.436, de 21 de setembro de 2017. Aprova a Política Nacional de Atenção Básica, estabelecendo a revisão de diretrizes para a organização da Atenção Básica, no âmbito do Sistema Único de Saúde (SUS). Diário oficial da União. 2017 set. 22; [acesso em 24 jun 2020]. Disponível em: https://bvsms.saude.gov.br/bvs/saudelegis/gm/2017/prt2436 2209 2017.html.

12. Nascimento WG do, Uchôa SA da C, Coêlho AA, Clementino F de S, Cosme MVB, Rosa RB, et al. Prescrição de medicamentos e exames por enfermeiros: contribuições à prática avançada e transformação do cuidado. Rev Latino-Am. Enfermagem. [Internet]. 2018 [acesso em 23 maio 2020]; 26:e3062. Disponível em: https://doi.org/10.1590/1518-8345.2423-3062.

13. Barbosa KTF, Costa KN de FM, Pontes M de L de F, Batista PS de S, Oliveira FMRL de, Fernandes $M$ das GM. Envelhecimento e vulnerabilidade individual: um panorama dos idosos vinculados à estratégia saúde da família. Texto contexto - enferm. [Internet]. 2017 [acesso em 23 maio 2020]; 26(2). Disponível em: https://doi.org/10.1590/0104-07072017002700015.

14. Fernandes MSSN, Cardoso AM. Educação em saúde como estratégia da enfermagem na disseminação de informações sobre a triagem neonatal às futuras mães. Revista Cien Escol Estad Saud Pubi - RESAP. [Internet]. 2018 [acesso em 23 maio 2020];4(1). Disponível em: http://www.revista.esap. go.gov.br/index.php/resap/article/view/67/83. 
COMO REFERENCIAR ESTE ARTIGO:

Pedrosa SC de S, Ferreira MAM, Guimarães KSL, Guimarães WFG, Barbosa KTF. Condutas que podem interferir na fase pré-analítica do exame sumário de urina. Cogitare enferm. [Internet]. 2021 [acesso em "colocar data de acesso, dia, mês abreviado e ano"]; 26. Disponível em: http://dx.doi.org/10.5380/ce.v26i0.74085.

Recebido em: 27/05/2020

Aprovado em: 10/12/2020

Editora associada: Luciana Alcântara Nogueira

Autor Correspondente:

Keylla Talitha Fernandes Barbosa

Centro Universitário de João Pessoa - João Pessoa, PB, Brasil

E-mail: keyllafernandes@gmail.com

Contribuição dos autores:

Contribuições substanciais para a concepção ou desenho do estudo; ou a aquisição, análise ou interpretação de dados do estudo - SCSP, MAMF, KTFB

Elaboração e revisão crítica do conteúdo intelectual do estudo - SCSP, KTFB

Aprovação da versão final do estudo a ser publicado - MAMF, KSLG, WFGG

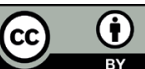

Copyright $\odot 2021$ Este é um artigo em acesso aberto distribuído nos termos da Licença Creative Commons Atribuição, que permite o uso irrestrito, a distribuição e reprodução em qualquer meio desde que o artigo original seja devidamente citado. 